PROCEEDINGS OF THE

AMERICAN MATHEMATICAL SOCIETY

Volume 134, Number 2, Pages 385-390

S 0002-9939(05)07796-8

Article electronically published on September 20, 2005

\title{
SPECTRAL RADIUS INEQUALITIES FOR HILBERT SPACE OPERATORS
}

\author{
FUAD KITTANEH
}

(Communicated by Joseph A. Ball)

\begin{abstract}
We prove several spectral radius inequalities for sums, products, and commutators of Hilbert space operators. Pinching inequalities for the spectral radius are also obtained.
\end{abstract}

\section{INTRODUCTION}

Let $B(H)$ denote the algebra of all bounded linear operators on a complex Hilbert space $H$. For $A \in B(H)$, let $r(A)$ and $\|A\|$ denote the spectral radius and the usual operator norm of $A$, respectively. It is well known that for every $A \in B(H)$, we have

$$
r(A) \leq\|A\|
$$

and that equality holds in the inequality (1) if $A$ is normal.

In addition to the inequality (1), the most important properties of the spectral radius are the spectral radius formula

$$
r(A)=\lim _{n \rightarrow \infty}\left\|A^{n}\right\|^{1 / n},
$$

a special case of the spectral mapping theorem, which asserts that

$$
r\left(A^{n}\right)=(r(A))^{n} \text { for every positive integer } n,
$$

and a commutativity property, which asserts that

$$
r(A B)=r(B A) \text { for every } A, B \in B(H) .
$$

The property (41) is an immediate consequence of the fact that the spectra of the operators $A B$ and $B A$ have the same nonzero elements.

It follows easily from the spectral radius formula (2) that if $A, B \in B(H)$ are such that $A B=B A$, then

$$
r(A+B) \leq r(A)+r(B)
$$

and

$$
r(A B) \leq r(A) r(B)
$$

Received by the editors March 17, 2004 and, in revised form, April 12, 2004.

2000 Mathematics Subject Classification. Primary 47A05, 47A10, 47A30, 47B47.

Key words and phrases. Spectral radius, inequality, operator matrix, commutator.

(C)2005 American Mathematical Society 
However, for noncommuting operators, the two-dimensional example $A=\left[\begin{array}{ll}0 & 1 \\ 0 & 0\end{array}\right]$, $B=\left[\begin{array}{ll}0 & 0 \\ 1 & 0\end{array}\right]$ shows that the spectral radius is neither subadditive nor submultiplicative. For additional properties of the spectral radius, the reader is referred to [1].

In Section 2 we establish a general spectral radius inequality, which yields spectral radius inequalities for sums and commutators of operators. Pinching inequalities for the spectral radius are also given. In Section 3 we present a spectral radius inequality for products of operators.

\section{A GenERAL SPECTRAL RAdiUs INEQUALity}

Our general spectral radius inequality is based on the property (4) and the following very useful lemma concerning $2 \times 2$ operator matrices, regarded as operators on $H \oplus H$. For generalizations and applications of this lemma, we refer to [2], 5], and the references therein.

Lemma 1. If $A, B, C, D \in B(H)$, then

$$
r\left(\left[\begin{array}{ll}
A & B \\
C & D
\end{array}\right]\right) \leq r\left(\left[\begin{array}{ll}
\|A\| & \|B\| \\
\|C\| & \|D\|
\end{array}\right]\right) .
$$

Now we are in a position to present our general spectral radius inequality.

Theorem 1. If $A_{1}, A_{2}, B_{1}, B_{2} \in B(H)$, then

$$
\begin{aligned}
r\left(A_{1} B_{1}+A_{2} B_{2}\right) \leq \frac{1}{2} & \left(\left\|B_{1} A_{1}\right\|+\left\|B_{2} A_{2}\right\|\right. \\
& \left.+\sqrt{\left(\left\|B_{1} A_{1}\right\|-\left\|B_{2} A_{2}\right\|\right)^{2}+4\left\|B_{1} A_{2}\right\|\left\|B_{2} A_{1}\right\|}\right) .
\end{aligned}
$$

Proof. We have

$$
\begin{aligned}
r\left(A_{1} B_{1}+A_{2} B_{2}\right)= & r\left(\left[\begin{array}{cc}
A_{1} B_{1}+A_{2} B_{2} & 0 \\
0 & 0
\end{array}\right]\right) \\
= & r\left(\left[\begin{array}{cc}
A_{1} & A_{2} \\
0 & 0
\end{array}\right]\left[\begin{array}{cc}
B_{1} & 0 \\
B_{2} & 0
\end{array}\right]\right) \\
= & \left.r\left(\left[\begin{array}{cc}
B_{1} & 0 \\
B_{2} & 0
\end{array}\right]\left[\begin{array}{cc}
A_{1} & A_{2} \\
0 & 0
\end{array}\right]\right) \quad \text { (by property (41) }\right) \\
= & r\left(\left[\begin{array}{ll}
B_{1} A_{1} & B_{1} A_{2} \\
B_{2} A_{1} & B_{2} A_{2}
\end{array}\right]\right) \\
\leq & r\left(\left[\begin{array}{ll}
\left\|B_{1} A_{1}\right\| & \left\|B_{1} A_{2}\right\| \\
\left\|B_{2} A_{1}\right\| & \left\|B_{2} A_{2}\right\|
\end{array}\right]\right) \quad(\text { by Lemma 1) } \\
= & \frac{1}{2}\left(\left\|B_{1} A_{1}\right\|+\left\|B_{2} A_{2}\right\|\right. \\
& \left.+\sqrt{\left(\left\|B_{1} A_{1}\right\|-\left\|B_{2} A_{2}\right\|\right)^{2}+4\left\|B_{1} A_{2}\right\|\left\|B_{2} A_{1}\right\|}\right)
\end{aligned}
$$

as required.

Theorem 1 includes several spectral radius inequalities as special cases. Among these inequalities, we obtain spectral radius inequalities for sums and commutators of operators. Pinching inequalities for the spectral radius are also obtained.

Corollary 1. If $A, B \in B(H)$, then

$$
r(A+B) \leq \frac{1}{2}\left(\|A\|+\|B\|+\sqrt{(\|A\|-\|B\|)^{2}+4 \min (\|A B\|,\|B A\|)}\right) .
$$


Proof. Letting $A_{1}=A, A_{2}=B_{1}=I$, and $B_{2}=B$ in Theorem 1 we have

$$
r(A+B) \leq \frac{1}{2}\left(\|A\|+\|B\|+\sqrt{(\|A\|-\|B\|)^{2}+4\|B A\|}\right) .
$$

By symmetry, it follows from inequality (10) that

$$
r(A+B) \leq \frac{1}{2}\left(\|A\|+\|B\|+\sqrt{(\|A\|-\|B\|)^{2}+4\|A B\|}\right) .
$$

The desired inequality (9) now follows from the inequalities (10) and (11).

Norm inequalities for sums of positive operators that are sharper than the triangle inequality and that are related to the inequality (9) have been recently given in [3. It should be mentioned here that the spectral radius in the inequality (9) cannot be replaced by the usual operator norm. To see this, consider $A=\left[\begin{array}{ll}1 & 1 \\ 1 & 1\end{array}\right]$ and $B=\left[\begin{array}{cc}1 & 1 \\ -1 & -1\end{array}\right]$. Then

$$
r(A+B)=\frac{1}{2}\left(\|A\|+\|B\|+\sqrt{(\|A\|-\|B\|)^{2}+4 \min (\|A B\|,\|B A\|)}\right)=2,
$$

but $\|A+B\|=2 \sqrt{2}$.

Recall that the commutator of the operators $A$ and $B$ is the operator $A B-B A$. The celebrated Kleinecke-Shirokov theorem says that if the commutator $A B-B A$ commutes with $A$, then $r(A B-B A)=0$. See, e.g., [1, p. 130]. The following two corollaries give estimates for $r(A B-B A)$ without any commutativity assumptions.

Corollary 2. If $A, B \in B(H)$, then

$$
r(A B \pm B A) \leq \frac{1}{2}\left(\|A B\|+\|B A\|+\sqrt{(\|A B\|-\|B A\|)^{2}+4\left\|A^{2}\right\|\left\|B^{2}\right\|}\right) .
$$

Proof. The inequality (12) follows from Theorem 1 by letting $A_{1}=B_{2}=A, B_{1}=$ $B$, and $A_{2}= \pm B$.

Corollary 3. If $A, B \in B(H)$, then

$$
r(A B \pm B A) \leq\|A B\|+\sqrt{\min \left(\|A\|\left\|A B^{2}\right\|,\|B\|\left\|A^{2} B\right\|\right)}
$$

and

$$
r(A B \pm B A) \leq\|B A\|+\sqrt{\min \left(\|A\|\left\|B^{2} A\right\|,\|B\|\left\|B A^{2}\right\|\right)} .
$$

Proof. Letting $A_{1}=I, A_{2}=B, B_{1}=A B$, and $B_{2}= \pm A$ in Theorem 1 we have

$$
r(A B \pm B A) \leq\|A B\|+\sqrt{\|A\|\left\|A B^{2}\right\|} .
$$

Similarly, letting $A_{1}=A B, A_{2}=B, B_{1}=I$, and $B_{2}= \pm A$ in Theorem 11, we have

$$
r(A B \pm B A) \leq\|A B\|+\sqrt{\|B\|\left\|A^{2} B\right\|} .
$$

The inequality (13) now follows from the inequalities (15) and (16). The inequality (14) follows from inequality (13) by symmetry.

In view of the unitary invariance and the submultiplicativity of the usual operator norm, utilizing either Corollary 2 or Corollary 3, we conclude the following spectral radius inequality, which can be used to derive pinching inequalities for the spectral radius. 
Corollary 4. If $A, U \in B(H)$ and $U$ is unitary, then

$$
r(A U \pm U A) \leq\|A\|+\left\|A^{2}\right\|^{1 / 2} .
$$

The pinching inequalities for the usual operator norm assert that if $A, B, C, D \in$ $B(H)$, then

$$
\left\|\left[\begin{array}{cc}
A & 0 \\
0 & D
\end{array}\right]\right\| \leq\left\|\left[\begin{array}{ll}
A & B \\
C & D
\end{array}\right]\right\|
$$

and

$$
\left\|\left[\begin{array}{cc}
0 & B \\
C & 0
\end{array}\right]\right\| \leq\left\|\left[\begin{array}{ll}
A & B \\
C & D
\end{array}\right]\right\| .
$$

It should be mentioned here that the usual operator norm in the inequalities (18) and (19) cannot be replaced by the spectral radius. To see this, consider $T=\left[\begin{array}{cc}1 & 1 \\ -1 & -1\end{array}\right]$. The inequality (17) enables us to obtain pinching inequalities for the spectral radius.

Theorem 2. If $A, B, C, D \in B(H)$, and if $T=\left[\begin{array}{c}A \\ C\end{array}\right]$

$$
\max (r(A), r(D)) \leq \frac{1}{2}\left(\|T\|+\left\|T^{2}\right\|^{1 / 2}\right)
$$

and

$$
\sqrt{r(B C)} \leq \frac{1}{2}\left(\|T\|+\left\|T^{2}\right\|^{1 / 2}\right) .
$$

Proof. Let $U=\left[\begin{array}{cc}I & 0 \\ 0 & -I\end{array}\right]$. Then $U$ is a unitary operator on $H \oplus H$,

$$
2\left[\begin{array}{cc}
A & 0 \\
0 & -D
\end{array}\right]=T U+U T,
$$

and

$$
2\left[\begin{array}{cc}
0 & -B \\
C & 0
\end{array}\right]=T U-U T \text {. }
$$

Thus

$$
\begin{aligned}
2 \max (r(A), r(D)) & =2 r\left(\left[\begin{array}{cc}
A & 0 \\
0 & D
\end{array}\right]\right) \\
& =r\left(\left[\begin{array}{cc}
2 A & 0 \\
0 & -2 D
\end{array}\right]\right) \\
& =r(T U+U T) \\
& \leq\|T\|+\left\|T^{2}\right\|^{1 / 2} \quad \text { (by Corollary 4) },
\end{aligned}
$$

and hence

$$
\max (r(A), r(D)) \leq \frac{1}{2}\left(\|T\|+\left\|T^{2}\right\|^{1 / 2}\right) .
$$

This proves the inequality (20).

To prove the inequality (21), first observe that $r\left(\left[\begin{array}{ll}0 & B \\ C & 0\end{array}\right]\right)=\sqrt{r(B C)}$. In fact, by the properties (3) and (4), we have

$$
\left(r\left(\left[\begin{array}{cc}
0 & B \\
C & 0
\end{array}\right]\right)\right)^{2}=r\left(\left[\begin{array}{cc}
0 & B \\
C & 0
\end{array}\right]^{2}\right)=r\left(\left[\begin{array}{cc}
B C & 0 \\
0 & C B
\end{array}\right]\right)=r(B C) .
$$


Thus

$$
\begin{aligned}
2 \sqrt{r(B C)} & =2 r\left(\left[\begin{array}{cc}
0 & B \\
C & 0
\end{array}\right]\right) \\
& =r\left(\left[\begin{array}{cc}
0 & -2 B \\
2 C & 0
\end{array}\right]\right) \\
& =r(T U-U T) \\
& \left.\leq\|T\|+\left\|T^{2}\right\|^{1 / 2} \quad \text { (by Corollary } 4\right),
\end{aligned}
$$

and hence

$$
\sqrt{r(B C)} \leq \frac{1}{2}\left(\|T\|+\left\|T^{2}\right\|^{1 / 2}\right) .
$$

This completes the proof of the theorem.

We remark that the inequalities (20) and (21) can also be obtained by employing Theorem 1 in [4, the numerical radius counterparts of the pinching inequalities (18) and (19), and the fact that the spectral radius of an operator is dominated by its numerical radius.

\section{A SPECTRAL RAdiUs INEQUALity FOR PRODUCTS OF OPERATORS}

Our spectral radius inequality for products of operators can be stated as follows.

Theorem 3. If $A, B \in B(H)$, then

$$
\begin{aligned}
r(A B) \leq \frac{1}{4} & (\|A B\|+\|B A\| \\
& \left.+\sqrt{(\|A B\|-\|B A\|)^{2}+4 \min (\|A\|\|B A B\|,\|B\|\|A B A\|)}\right) .
\end{aligned}
$$

Proof. We have

$$
\begin{aligned}
2 r(A B) & =r\left(\left[\begin{array}{cc}
2 A B & 0 \\
B & 0
\end{array}\right]\right) \quad(\text { see Problem } 71 \text { in [1] }) \\
& =r\left(\left[\begin{array}{cc}
I & A \\
0 & I
\end{array}\right]\left[\begin{array}{cc}
A B & 0 \\
B & 0
\end{array}\right]\right) \\
& =r\left(\left[\begin{array}{cc}
A B & 0 \\
B & 0
\end{array}\right]\left[\begin{array}{cc}
I & A \\
0 & I
\end{array}\right]\right) \quad(\text { by property (44) }) \\
& =r\left(\left[\begin{array}{cc}
A B & A B A \\
B & B A
\end{array}\right]\right) \\
& \leq r\left(\left[\begin{array}{cc}
\|A B\| & \|A B A\|] \\
\|B\| & \|B A\|
\end{array}\right]\right) \quad(\text { by Lemma 10) } \\
& =\frac{1}{2}\left(\|A B\|+\|B A\|+\sqrt{(\|A B\|-\|B A\|)^{2}+4\|B\|\|A B A\|}\right),
\end{aligned}
$$

and hence

$$
r(A B) \leq \frac{1}{4}\left(\|A B\|+\|B A\|+\sqrt{(\|A B\|-\|B A\|)^{2}+4\|B\|\|A B A\|}\right) .
$$

By symmetry and the property (4), it follows from the inequality (23) that

$$
r(A B) \leq \frac{1}{4}\left(\|A B\|+\|B A\|+\sqrt{(\|A B\|-\|B A\|)^{2}+4\|A\|\|B A B\|}\right) .
$$

The inequality (22) now follows from the inequalities (23) and (24). 
We remark that an alternative proof of Theorem 3 can be based on Theorem 1 Finally, we remark that the spectral radius inequalities presented in this paper have diverse applications. For example, it is possible to obtain new bounds for the zeros of monic polynomials by applying some of these spectral radius inequalities to the Frobenius companion matrices of these polynomials. For relevant analysis, we refer the interested reader to [4, 5], and the references therein.

\section{ACKNOWLEDGMENT}

The author is grateful to the referee for his comments.

\section{REFERENCES}

1. P. R. Halmos, A Hilbert Space Problem Book, 2nd ed., Springer-Verlag, New York, 1982. MR0675952(84e:47001)

2. J. C. Hou and H. K. Du, Norm inequalities of positive operator matrices, Integral Equations Operator Theory 22 (1995), 281-294. MR1337376 (96e:47007)

3. F. Kittaneh, Norm inequalities for sums of positive operators, J. Operator Theory 48 (2002), 95-103. MR1926046 (2003g:47016)

4. F. Kittaneh, A numerical radius inequality and an estimate for the numerical radius of the Frobenius companion matrix, Studia Math. 158 (2003), 11-17. MR2014548 (2004i:15022)

5. F. Kittaneh, Bounds for the zeros of polynomials from matrix inequalities, Arch. Math. (Basel) 81 (2003), 601-608. MR2029723 (2004j:15035)

Department of Mathematics, University of Jordan, Amman, Jordan

E-mail address: fkitt@ju.edu.jo 